\title{
THE CONJUGATION REPRESENTATION AND FUSIONLESS EXTENSIONS
}

\author{
EDWARD FORMANEK
}

Abstract. Let $G$ be a finite group with center $Z$. $G$ acts on itself by conjugation, and this induces a permutation representation $P$ of $G / Z$. It has been conjectured that every complex irreducible representation of $G / Z$ occurs in $P$. This paper gives a counterexample to this conjecture.

Let $G$ be a finite group with center $Z$. $G$ acts on itself by conjugation and this action yields a permutation representation $P$ of $G . P$ is in fact a representation of $G / Z$, and this motivates the following question, asked by Richard Roth [2]:

Does every complex irreducible representation of $G / Z$ occur in $P$ ?

The answer is no, and the purpose of this paper is to give a counterexample.

Lemma 1. Suppose $R$ is a one-dimensional representation of $G$ with kernel $K$. Then $R$ occurs in $P$ iff $K$ contains the centralizer of some element of $G$.

Proof. Let $\left\{g_{1}\right\}, \cdots,\left\{g_{n}\right\}=$ conjugacy classes of $G ; H_{i}=$ centralizer of $g_{i} ; P_{i}=$ permutation representation of $G$ afforded by the action of $G$ on $\left\{g_{i}\right\}=$ representation of $G$ induced by the principal representation $X_{i}$ of $H_{i}$.

$$
P=P_{1}+\cdots+P_{n} \quad \text { and } \quad\left(R \mid H_{i}, X_{i}\right)_{H_{i}}=\left(R, P_{i}\right)_{G}
$$

by Frobenius reciprocity. Thus $R$ occurs in $P$ (and hence in some $P_{i}$ ) iff $K$ contains some $H_{i}$ (i.e. $R \mid H_{i}=X_{i}$ ).

Suppose that $\phi$ is an outer automorphism of a group $K$ and that $\phi$ has order $r$ as an outer automorphism (i.e. $\phi^{r}$ is inner, but no smaller power of $\phi$ is inner). If $\phi^{r}$ acts like conjugation by $a$ in $K$, we will let $(K, \phi, a)$ denote the group $\left\{K, x: x^{r}=a, k^{x}=\phi(k)\right.$ for all $\left.k \in K\right\}$.

Lemma 2. Suppose that $G=(K, \phi, a)$ is defined as above, and that $\phi$ carries each conjugacy class of $K$ into itself (i.e. $G$ is a fusionless extension of $K)$. Let $R$ be a one-dimensional complex representation of $G$ with kernel $K$. Then $R$ is a representation of $G / Z$, but $R$ does not occur in the conjugation representation $P$ of $G$.

Received by the editors February 1, 1971.

AMS 1969 subject classifications. Primary 2020, 2080.

Key words and phrases. Finite group, conjugation representation, permutation representation. 
Proof. $K$ contains the center $Z$ of $G$ since $\phi$ is outer. $K$ does not contain the centralizer of any element of $G$ since $\phi$ leaves invariant the conjugacy classes of $K$. Thus $R$ does not occur in $P$, by Lemma 1 .

By virtue of Lemma 2 we need only observe that there are finite groups with class preserving outer automorphisms. The first examples were given by Burnside [1].

\section{REFERENCES}

1. William Burnside, On the outer automorphisms of a group, Proc. London Math. Soc. (2) 11 (1913), 40-42.

2. Richard Roth, On the conjugating representation of a finite group, Pacific J. Math. 37 (to appear).

University OF MisSOURI-St. Louis, St. LouIs, Missouri 63121 